\title{
Audio Scenarios Detection Technique
}

\author{
Ajay Kadam \\ Student: Department of Computer Engineering \\ Dr. D. Y. Patil School of Engineering and \\ Technology \\ Lohegaon, Pune, India
}

\author{
Ramesh M. Kagalkar \\ Professor: Department of Computer Engineering \\ Dr. D. Y. Patil School of Engineering and \\ Technology \\ Lohegaon, Pune, India
}

\begin{abstract}
The proposed research objective is to add to a framework for programmed recognition of sound. In this framework the real errand is to distinguish any information sound stream investigate it \& anticipate the likelihood of diverse sounds show up in it. To create and industrially conveyed an adaptable sound web crawler a flexible sound search engine. The calculation is clamor and contortion safe, computationally productive, and hugely adaptable, equipped for rapidly recognizing a short portion of sound stream caught through a phone microphone in the presence of frontal area voices and other predominant commotion, and through voice codec pressure, out of a database of over accessible tracks. The algorithm utilizes a combinatorial hashed time-recurrence group of stars examination of the sound, yielding ordinary properties, for example, transparency, in which numerous tracks combined may each be distinguished.
\end{abstract}

\section{Keywords}

Finger printing, Pure tone, White noise

\section{INTRODUCTION}

The human movement is reflected in a rich mixture of acoustic occasions, delivered either by the human or by articles took care of by people, so the determination of both the character of sounds and their position in time can help identify and depict that human action. The improvement of frameworks for programmed grouping of a sound sign into classes of occasions, or the change of the exhibitions of programmed arrangement of a sound sign into occasions[1].

In proposed framework we are going to give the info as a sound stream then after preprocessing and highlight extraction sound stream is perceived and showed on the showcase that is brought from database which is now put away \& right stable class is distinguished and showed on the screen. The example coordinating and acknowledgment could be possible through predefined database.

Generally to activate verbalization signal capture for verbalization apperception, the push-to-verbalize has been widely utilized in handheld mobile contrivances in which one can push a special button in the contrivance to activate or deactivate verbalization capture[6]. It is immune to the environmental noise, but may not the case virtually required in other consumer contrivances; for example, an interactive digital TV is supposed to heedfully auricularly discern all the time and automatically detect only human voice by denotes of the hands-free verbalization acquisition. In integration, it requires filtering out potential acoustic event sounds (e.g., hand-claps, phone ringing, door-slam, and so on) for the robustness of speech recognition system [5].

Not at all like other other audio or verbalization signals, sound events have a generally brief time compass. They are generally recognized by their exceptional spectra-fleeting mark. This paper proposes a novel order technique taking into account probabilistic separation bolster vector machines (SVMs). We concentrate on a parametric way to deal with describing sound signs utilizing the dissemination of the sub band fleeting envelope (STE), and part methods for the sub band probabilistic separation (SPD) under the system of SVM. We demonstrate that summed up gamma displaying is very much contrived for sound portrayal and that the probabilistic separation bit gives a shut structure answer for the figuring of difference separation, which colossally lessens computational expense. We directed investigations on a database of ten sorts of sound occasions. The outcomes demonstrate that the proposed characterization technique altogether beats traditional SVM classifiers with Mel-recurrence campestral coefficients (MFCCs). The quick processing of probabilistic separation likewise makes the proposed technique a conspicuous decision for online sound occasion acknowledgment [2].

We think about the spectral and transient periodicity representations that can be utilized to portray the qualities of the mood of a music sound sign. A nonstop esteemed vitality capacity speaking to the onset positions after some time is initially separated from the sound sign. From this capacity we register at every time a vector which speaks to the attributes of the nearby mood. Four capabilities are concentrated on for this vector. They are gotten from the plentifulness of the discrete Fourier change (DFT), the auto-relationship capacity (ACF), the result of the DFT and the ACF interjected on a half and half slack/recurrence hub and the connected DFT and ACF coefficients. At that point the vectors are inspected at some particular frequencies, which speak to different proportions of the neighborhood beat. The capacity of these periodicity representations to depict the musicality attributes of a sound thing is assessed through an arrangement errand. In this, we test the utilization of the periodicity representations alone, joined with rhythm data and consolidated with a proposed arrangement of cadence highlights. The assessment is performed utilizing commented and assessed rhythm. We demonstrate that utilizing such straightforward periodicity representations permits accomplishing high acknowledgment rates at any rate equivalent to beforehand distributed results [3].

A music piece can be considered as an arrangement of sound occasions which speak to both transient and long haul fleeting data. Nonetheless, in the assignment of programmed music class characterization, the majority of content order based methodologies could just catch fleeting neighbourhood conditions (e.g., unigram and bigram-based event insights) to speak to music substance. In this paper, we propose the utilization of time-obliged consecutive examples (TSPs) as compelling highlights for music classification arrangement[12]. Above all else, a programmed dialect distinguishing proof method is performed to tokenize every music piece into an 
arrangement of shrouded Markov model files. At that point TSP mining is connected to find classification particular TSPs, trailed by the reckoning of event. Frequencies of TSPs in every music piece[15][21]. At last, bolster vector machine classifiers are utilized in view of these event frequencies to perform the arrangement assignment. Examinations led on two generally utilized datasets for music kind characterization, GTZAN and ISMIR2004Genre, demonstrate that the proposed strategy can find more discriminative fleeting structures and attain to a superior acknowledgment precision than the unigram and bigram-based measurable methodology [4].

\section{FUNDAMENTAL APPROACH}

1. Beforehand, our application fingerprints a comprehensive catalog of recordings, also, stores the fingerprints in a database.

2. A client "labels" a recording we listen, which fingerprints a 10 second example of audio.

3. Our app uploads the fingerprint to Web administration, which runs a quest for a coordinating unique finger impression in their database.

4. In the event that a match is found, the Record data is come back to the client, generally a lapse is returned.

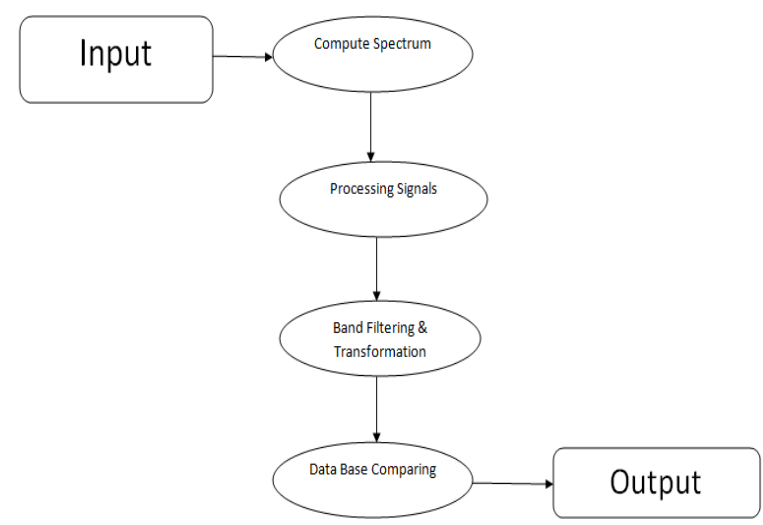

Figure 1: Block diagram of system

\section{FINGERPRINTING PROCEDURE}

The system can think about any bit of music as a period recurrence chart called a spectrogram. On one hub is time, on another is recurrence, and on the 3rd is force. Every point on the chart speaks to the force of a given recurrence at a particular point in time. Accepting time is on the x-pivot and recurrence is on the y-hub, an even line would speak to a ceaseless unadulterated tone and a vertical line would speak to an immediate blast of repetitive sound[16][19][22].

The algorithm fingerprints a song by generating this data is come back to the client, generally a lapse is returned.

Table 1: Peak intensities storage

\begin{tabular}{|c|c|}
\hline Frequency in $\mathrm{MHz}$ & Time in sec \\
\hline 823.44 & 1.054 \\
\hline 11892.31 & 1.321 \\
\hline 712.84 & 1.703 \\
\hline$\ldots$ & $\ldots$ \\
\hline 819.71 & 9.943 \\
\hline
\end{tabular}

Application constructs their unique finger impression index out as a hash table, where the key is the recurrence. At the point when our application gets a finger impression like the one above, it utilizes the first key (for this situation 823.44), and it hunt down every coordinating melody. Their hash table may look like after.

Table 1.2: Fingerprint mapping with hash

\begin{tabular}{|c|c|}
\hline Frequency in $\mathrm{MHz}$ & Time in sec \& song information \\
\hline 823.44 & 1.054 song $\mathrm{A}$ by artist 1 \\
\hline 11892.31 & 1.321 song B by artist 2 \\
\hline 712.84 & 1.703 song $\mathrm{c}$ by artist 2 \\
\hline$\ldots$ & $\ldots$ \\
\hline 819.71 & 9.943 song $X$ by artist 2 \\
\hline
\end{tabular}

We not only mark a solitary point in the spectrogram, rather we mark a dyad of focuses: the "peak intensity" in addition to a second "anchor point". So their key is not only a solitary recurrence, it is a hash of the frequencies of both focuses. This prompts less hash crashes which thusly expedites catalog probing by several orders of magnitude by sanctioning them to take more preponderant advantage of the table's constant (O(1)) look-up Time[18][20].

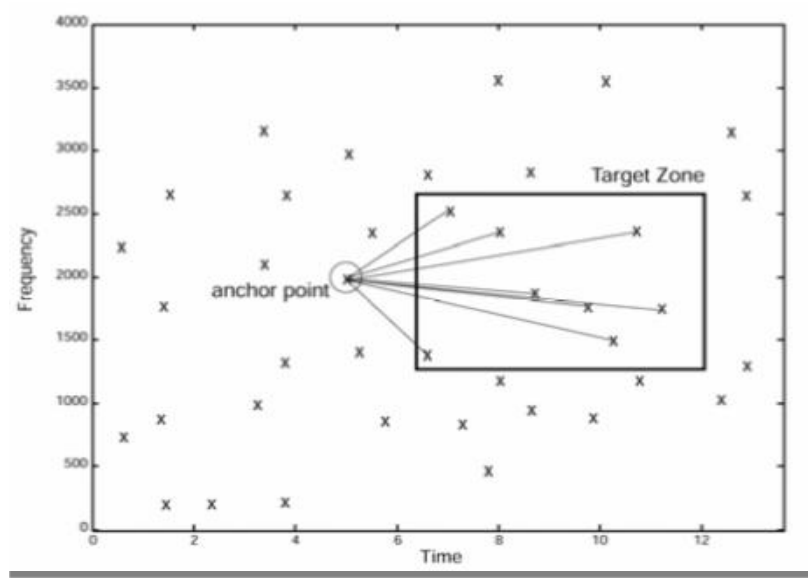

Figure 2 : Histogram of a non-matching run

First we engender a spectrogram for the musical composition. The spectrogram is a 3 measurements diagram: on the even $(\mathrm{X})$ pivot, you have the on the (Y) pivot you have the recurrence and the third measurement is spoken to by shading force and it speaks to the plentifulness of a certain recurrence. Thus fundamentally, a spot on the chart will speak to the volume of a certain sound at a certain time in the musical composition. A more tenebrous point denotes that the categorical sound (recurrence) is louder than a lighter point [17].

Putting away the full musical composition in the database will occupy a gargantuan amount of space, considering that they database has many musical compositions. So instead, we will store only the excruciating sounds in the musical composition, the time when they appear in the musical composition and at which frequency

Now, we will draw an incipient chart called dissipate diagram. The diagram will have on the even pivot $(\mathrm{X})$ the season of the musical composition in the database and on the vertical pivot (Y) the season of the recorded specimen. On the $\mathrm{X}$ pivot we will check th2 and on the $\mathrm{Y}$ hub we will stamp th1. The purpose of crossing point of the two event times (th1 and th2) 
will be checked with a diminutive circle. The magic transpires now: if the graph will contain an abundance of pairs of th1's and th2 s from the same musical composition, a diagonal line will compose. The conception abaft the formation of that line is straightforward: the rate at which the crests (the minuscule crosses from the improved spectrogram) in the database musical composition appear will be identically tantamount rate in which the tops show up in the recorded specimen, so on the off chance that you match these circumstances, the directions on the dissipate diagram will develop perpetually (to one side top of the diagram) as the time goes on both tomahawks [9][10][13].

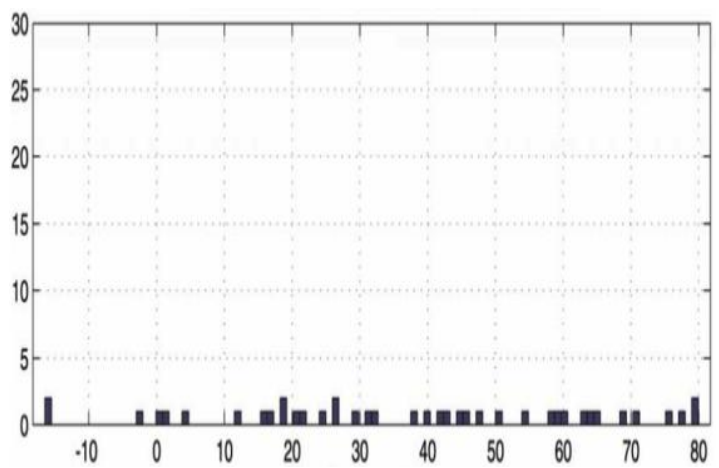

Figure 3: Histogram of a non-matching run.

At long last, we will compute a contrast in the middle of th2 and th1 (dth) and we will plot it in a histogram. In the event that there is a match in the chart plotted, then there will be a great deal of dths with the same quality, on the grounds that, essentially, subtracting the th2 from th1 will give the counterbalance from where the specimen was recorded (the distinction between a point in the pristine musical composition also, the same point in the recorded sample[8][14]. This will bring about a top inside of the histogram, which will attest a match Probability $\mathrm{p}$ of survival for all points involved, then the probability of at least one hash surviving per anchor point is

$\mathrm{p}^{*}\left[1-(1-\mathrm{p})^{\wedge} \mathrm{F}\right.$

For reasonably large values of $\mathrm{F}$, e.g. F>10 \& reasonable values of $p$,

E.g. $p>0.1$, we have approximately

$\mathrm{p}=\mathrm{p} *\left[1-(1 * \mathrm{p})^{\wedge} \mathrm{F}\right]$

$\mathrm{F}$ is frequency

Searching and sorting

The following technique solves the problem in approximately $\mathrm{N} * \log (\mathrm{N})$ time,

where $\mathrm{N}$ is the number of points appearing on the scatterplot $t^{\prime}=$ t+offset

$t^{\prime}$ is time co ordinate of the feature in the matching database

$t$ is time coordinate of the corresponding feature in the sample

sound file to be identified offset is the approx number generated

randomly

\section{FINDING OF SONGS BASED ON THE RECORDED SAMPLE}

Well, we first and foremost rehash the same fingerprinting likewise to the recorded example. Every hash produced from the specimen sound, will be scanned for a match in the database [7][11].

On the off chance that a match is discovered you will have the season of the hash from the specimen (th1), the season of the hash from the musical composition in the database (th2) and verifiably the ID of the of the musical composition for which the hash matched. Fundamentally, th1 is the time since the commencement of the specimen until the season of the example hash and th2 is the time subsequent to the commencement of the musical composition and the time of the musical composition hash.

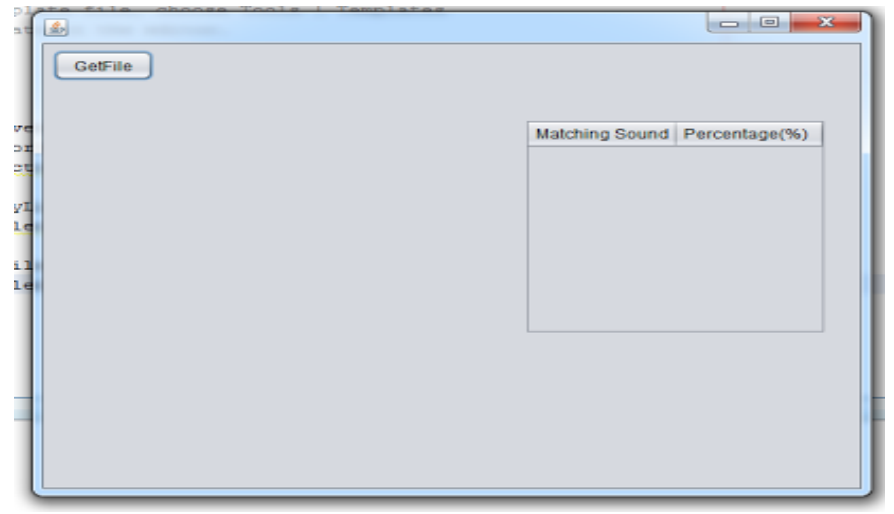

Figure 4: Implementation results.

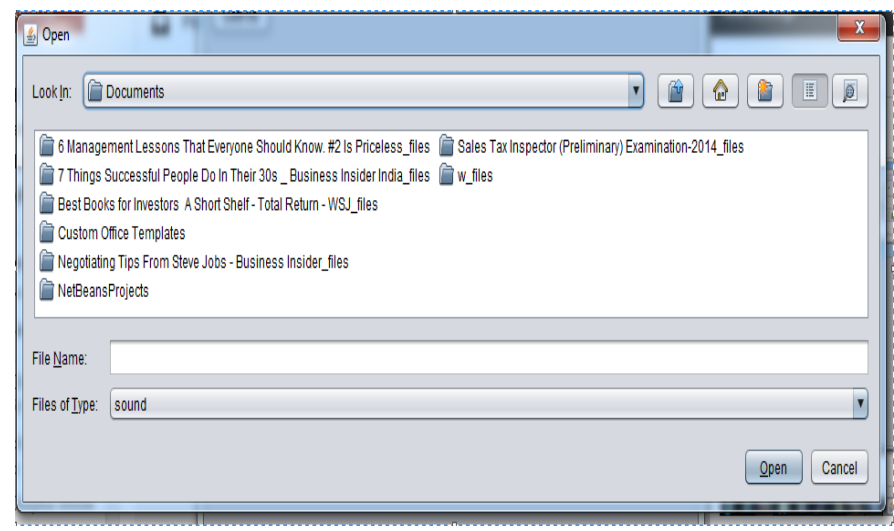

Figure5 : Implementation results.

\section{CONCLUSIONS}

The proposed framework gives hands on specialized apparatus to expand and to discover the classes of not the same as its sound example of a specimen space. This is conceivable by our proposed methodology. In this methodology information base is made, which comprises of .wave type of distinctive movement, occasion, scenes sounds what's more, its data of every example model of different sorts. When the sound example question is given to the framework first it changes over into .wave design and concentrate highlights. Every stable document is "fingerprinted," a procedure in which reproducible hash tokens are separated. Both "database" and "test" sound documents are subjected to the same examination. The fingerprints from the obscure example are coordinated against a substantial arrangement of fingerprints got from the test database. The framework matches are consequently assessed for rightness of match.

\section{ACKNOWLEDGMENT}

I would like to thank the researchers as well as publishers for making their resources available and teachers for their guidance. The authors would like to thank Chairman D.Y.Patil 
and Management and the Director/Principal Dr. Uttam Kalwan, Head, Coordinator, Guide, and Colleagues of the Department of Computer Engineering, Dr.D.Y.Patil School of Engineering and Technology, Charoli, B.K.Via -Lohegaon, Pune, Maharstra, India, for their support, suggestions and encouragement.

\section{REFERENCES}

[1] Ajay R.Kadam \& Ramesh Kagalkar "Predictive Sound Recognization System" International Journal of Advance Research in Computer Science and Management Studies Volume 2, Issue 11, November 2014.

[2] Namgook Cho \& Eun-Kyoung Kim "Enhanced-voice activity detection using acoustic event detection \& classification" in IEEE Transactions on Consumer Electronics, Vol. 57, No. 1, February 2011.

[3] Shivaji Chaudhari and Ramesh Kagalkar "A Review of Automatic Speaker recognization and Identifying Speaker Emotion Using Voice Signal" International Journal of Science and Research (IJSR), Volume 3, Issue 11 November 2014.

[4] Geoffroy Peeters"spectral and temporal periodicity representations of rhythm," ieee transactions on audio, speech, and language processing, vol. 19, no. 5, july 2011

[5] Jia-Min Ren, Student Member, IEEE, and Jyh-Shing Roger Jang, Member, IEEE "discovering time constrained sequential patterns for music genre classification" IEEE transactions on audio, speech, and language processing, vol. 20, no. 4, may 2012.

[6] Namgook Choo \& Taeyoon Kim "Voice activation system using acoustic event detection and keyword/speaker recognition" 01/2011; DOI: 10.1109/ICCE.2011.5722550

[7] G. Valenzise, L. Gerosa, M. Tagliasacchi, F. Antonacci, and A. Sarti, "Scream and gunshot detection and localization for audio-surveillance systems," in Proc. IEEE Conf. Adv. Video Signal Based Surveill., 2007, pp. 21-26.

[8] Kyuwoong Hwang and Soo-Young Lee, Member, IEEE "Environmental Audio Scene and Activity Recognitionthrough Mobile-based Crowdsourcing" IEEE Transactions on Consumer Electronics, Vol. 58, No. 2, May 2012.

[9] R. Radhakrishnan, A. Divakaran, and P. Smaragdis, "Audio analysis for surveillance applications," in Proc. IEEE Workshop Applicat. Signal Process. Audio Acoust., 2005, pp. 158-161.

[10] Proc. (RT-07) Rich Transcription Meeting Recognition Evaluation Plan, [Online]. Available: http://www.nist.gov/speech/tests/rt/rt2007

[11] J. Tchorz and B. Kollmeier, "A model of auditory perception as front end for automatic speech recognition," J. Acoust. Soc. Amer., vol. 106, no. 4, pp. 2040-2050, 1999.

[12] T. Jaakkola and D. Haussler, "Exploiting generative models in discriminative classifiers," in Advances in Neural Information Processing Systems. Cambridge, MA: MIT Press, 1998, vol. 11, pp. 487-493.

[13] V. Wan and S. Renals, "Speaker verification using sequence discriminant support vector machines," IEEE
Trans. Speech Audio Process., vol. 13, no. 2, pp. 203-210, Mar. 2005.

[14] T. Jebara and R. Kondor, "Bhattacharyya and expected likelihood kernels," Lecture Notes in Computer Science, vol. 2777, pp. 57-71, 2003.

[15] W. M. Campbell, D. E. Sturim, and D. A. Reynolds, "Support vector machines using GMM pervectors for speaker verification," IEEE Signal Process. Lett., vol. 13, no. 5,pp. 308-311, May 2006.

[16] Abeer Alwan, Steven Lulich, Harish Ariskere "The role of subglottal resonances in speech processing algorithms" The Journal of the Acoustical Society of America (Impact Factor:1.56). 04/2015;137(4):2327-2327.

[17] Fred Richardson, Douglas Reynolds, Najim Dehak “Deep Neural Network Approaches to Speaker and Language Recognition" IEEE Signal Processing Letters (Impact Factor: 1.64). 10/2015; 22(10):1-1

[18] Jens Kreitewolf, Angela D Friederici, Katharina von Kriegstein "Hemispheric Lateralization of Linguistic Prosody Recognition in Comparison to Speech and Speaker Recognition."NeuroImage (Impact Factor: 6.13). 07/2014;102DOI: 10.1016/j.neuroimage.2014.07.0

[19] Kun Han, Yuxuan Wang, DeLiang Wang, William S. Woods, Ivo Merks, Tao Zhang "Learning Spectral Mapping for Speech Dereverberation and Denoising" Audio, Speech, and Language Processing, IEEE/ACM Transactions on 06/2015; 23(6):982-992. DOI: $10.1109 /$ TASLP.2015.2416653

[20] Mikolaj Kundegorski, Philip J.B. Jackson, Bartosz Ziółko" Two-Microphone Dereverberation for Automatic Speech Recognition of Polish" Archives of Acoustics 01/2015; 39(3). DOI: 10.2478/aoa-2014-0045

[21] Kaveri Kamble and Ramesh Kagalkar "Audio Visual Speech Synthesis and Speech Recognition for Hindi Language" International Journal of Computer Science and Information Technologies, Vol. 6 (2) , 2015, 17791783 .

[22] Abeer Alwan, Steven Lulich, Harish Ariskere" The role of subglottal resonances in speech processing algorithms" The Journal of the Acoustical Society of America (Impact Factor: $\quad 1.56) .04 / 2015 ; \quad 137(4): 2327-2327$. DOI: $10.1121 / 1.4920497$

\section{AUTHORS PROFILE}

\section{Ajay Kadam}

Mr. Ajay Kadam received Bachelor of Engineering degree in Computer Science \& Engineering in ........... and now pursuing Post Graduation (M.E.) in department of Computer Engineering from Dr. D.Y.Patil School of Engineering and Technology in current academic year 2014-15. He I currently working on audio sound reorganization.

\section{Ramesh. M. Kagalkar}

Ramesh. M. Kagalkar was born on Jun 1st, 1979 in Karnataka, India and presently working as Assistant. Professor, Department of Computer Engineering, Dr.D.Y.Patil School Of Engineering and Technology, Charoli, B.K.Via -Lohegaon, Pune, Maharashtra, India. He has 13.8 years of teaching experience at various institutions. He is a Research Scholar in Visveswaraiah Technological University, Belgaum, He had obtained M.Tech (CSE) Degree in 2005 from VTU Belgaum and $\mathrm{He}$ received BE (CSE) Degree in 2001 from Gulbarga 
University, Gulbarga. He is the author of text book Advance Computer Architecture, which cover the syllabus of final year computer science and engg department, Visveswaraiah Technological University, Belgaum. $\mathrm{He}$ is waiting for submission of two research articles for patent right. He has published more than 18 research papers in International Journals and presented few of there in international conferences. His main research interest include Image processing, Gesture reorganization, speech processing, voice to sign language and CBIR. Under his guidance two ME students awarded degree in SPPU, Pune, two more students at the edge of completion their ME final dissertation reports and 5 final year M.E students are started a research work on different area and they have publish their research papers on International Journals and International conference. 2 ME Ist year students are started a research work on different area. 